\title{
Diffusion-Weighted Single-Shot Line Scan Imaging of the Human Brain
}

\author{
Jürgen Finsterbusch and Jens Frahm*
}

\begin{abstract}
Single-shot line scan imaging (LSI) was adapted to diffusionweighted (DW) MRI by replacing the initial $90^{\circ}$ radiofrequency pulse of the underlying high-speed stimulated echo sequence by a DW spin-echo preparation period. Implementation on a 2.0 T whole-body MRI system yielded DW images of the human brain with $b$ factors of $750 \mathrm{~s} \mathrm{~mm}^{-2}$ and total imaging times of about $500 \mathrm{~ms}$ either for a single slice at $1.5 \times 3.0 \times 6 \mathrm{~mm}^{3}$ resolution or simultaneously for up to seven slices at $3.75 \times$ $3.75 \times 8 \mathrm{~mm}^{3}$ resolution. Isotropic DW images and maps of the trace of the diffusion tensor were calculated from four scans with different combinations of three orthogonal diffusion gradients. DW LSI combines high speed with robustness against image artifacts caused by motion (no phase ghosting) and tissue susceptibility differences (no signal losses, no geometric distortions). Because the latter is an important advantage over echo-planar imaging, DW LSI may find useful applications despite a limited signal-to-noise ratio. Magn Reson Med 42:772778, 1999. 시 1999 Wiley-Liss, Inc.
\end{abstract}

Key words: magnetic resonance imaging; high-speed imaging; diffusion-weighted imaging; apparent diffusion coefficient

Particularly in acute stroke $(1,2)$, the assessment of water proton diffusion gains increasing importance as a sensitive tool for a functional characterization of human brain tissue. Technically, diffusion-weighted (DW) magnetic resonance imaging (MRI) is adversely affected by the presence of unavoidable macroscopic movements due to involuntary head motion, breathing, and cardiac-related tissue pulsations. Pertinent phase distortions are amplified in the presence of strong diffusion gradients and translate into ghosting artifacts in Fourier images along the direction of the phase-encoding gradient. To overcome this problem, most recent applications of DW MRI are based on echoplanar imaging (EPI) in which diffusion-induced phase twists are constant for all gradient echoes and therefore do not affect the calculation of a magnitude image (3). Furthermore, the high speed of the EPI acquisition minimizes motion effects during the gradient echo train.

Notwithstanding these advantages, EPI suffers severe geometric image distortions and even total signal losses in brain regions where large differences in tissue magnetic susceptibility cause local magnetic field inhomogeneities. These problems may be either minimized by the acquisition of gradient echoes at very short echo times as in subsond FLASH (4) or completely avoided by generating $\mathrm{RF}$ refocused echoes as in high-speed STEAM (5) and RARE (6). However, whereas DW high-speed STEAM (7) and DW subsond FLASH $(8,9)$ exhibit a rather limited

Biomedizinische NMR Forschungs GmbH am, Max-Planck-Institut für biophysikalische Chemie, Göttingen, Germany.

${ }^{*}$ Correspondence to: Jens Frahm, Ph.D., Biomedizinische NMR Forschungs $\mathrm{GmbH}$ am, Max-Planck-Institut für biophysikalische Chemie, Am Fassberg11, D-37070 Göttingen, Germany. E-mail: jrahm@gwdg.de

Received 26 March 1999; revised 18 June 1999; accepted 2 July 1999.

(c) 1999 Wiley-Liss, Inc. signal-to-noise ratio (SNR) due to the use of low flip angle excitations, DW RARE (10) results in a special form of motion sensitivity that only vanishes after a combination of separately acquired magnitude images from the two underlying echo paths (11).

Line scan imaging (LSI) based on RF refocused echoes $(12,13)$ has a lower SNR than Fourier imaging but does not rely on spatial phase encoding. Thus, LSI is inherently insensitive to all mechanisms causing a variation of the MRI signal phase. Recently, a rapid LSI technique (14) has been adapted to DW MRI $(15,16)$. The approach employs a cross-sectional spin-echo (SE) excitation of a columnar intersection volume or "line" that is spatially shifted to obtain a complete image within typical measuring times of approximately 20-30 s.

The purpose of this work is the development of a DW variant of a single-shot high-speed LSI technique based on stimulated echoes (17). It combines the robustness of LSI against motion artifacts and susceptibility problems with subsecond acquisition times even without a gradient performance as required for EPI. Furthermore, the new LSI method is capable of measuring multiple slices within a single shot and thus offers a user-selectable trade-off between spatial resolution, field-of-view (FOV), and volume coverage.

\section{MATERIALS AND METHODS}

\section{Basic Pulse Sequence for DW Single-Shot LSI}

Figure 1 shows the basic pulse sequence for DW singleshot LSI using stimulated echo (STE) signals. The example refers to a simultaneous multi-slice acquisition of three slices. Spoiler gradients are omitted for the sake of simplicity. With reference to the sequence versions developed for high-speed LSI in (17), the present variant employs a nonselective SE preparation phase to allow for a simultaneous DW of all slices. This $90^{\circ}-180^{\circ} \mathrm{RF}$ excitation and refocusing period symmetrically encompasses three orthogonal diffusion gradients (for details see below). It corresponds to the first $90^{\circ} \mathrm{RF}$ excitation pulse of a conventional STEAM sequence with the sond half of the SE signal replacing the normal FID. Because of the temporal decoupling of DW and high-speed LSI, cross-product terms between the imaging and diffusion gradients are avoided. This particularly applies to the predephasing read gradient lobes that are required between the slice-selective $90^{\circ} \mathrm{RF}$ pulses in the sond interval (left bracket in Fig. 1) to shift the echo times of the respective line-selective STE signals during repetitive data acquisition (right bracket in Fig. 1).

It should be noted that different slices have different TE values, i.e., $\mathrm{T}_{2}$ weighting, and different lines within the same slice have different $\mathrm{TM}$ values, i.e., $\mathrm{T}_{1}$ weighting. 


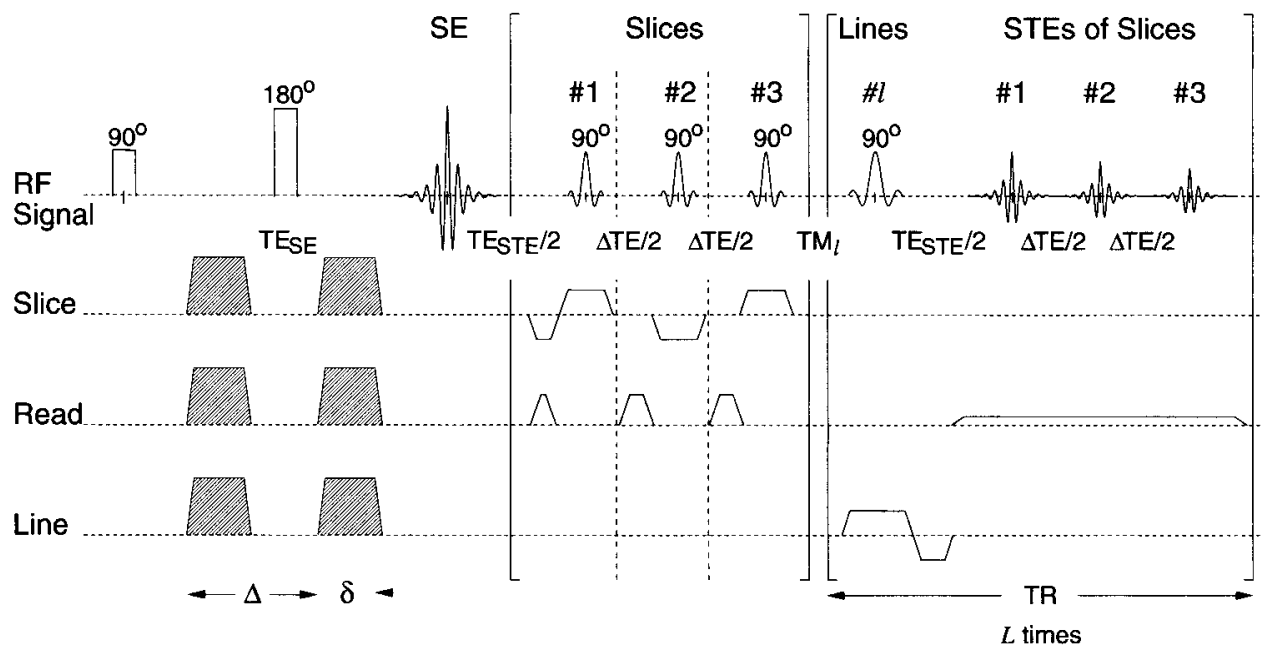

FIG. 1. Basic MRI sequence for diffusion-weighted single-shot line scan imaging (DW LSI) using stimulated echoes (STE). The example refers to a multi-slice version for the simultaneous acquisition of three slices. The leading part represents a nonselective DW spin echo preparation period. The left bracket excites the desired slices by generating temporally shifted longitudinal magnetizations. The right bracket refers to the repetitive readout of $L$ spatially shifted lines with increasing middle intervals $\mathrm{TM}_{\mathrm{I}}$. For each line the STE signals at echo times $\mathrm{TE}_{\mathrm{STE}}, \mathrm{TE}_{\mathrm{STE}}+\Delta \mathrm{TE}$, and $\mathrm{TE}_{\mathrm{STE}}+2 \times \Delta \mathrm{TE}$ correspond to the excited slices.

Whereas the $\mathrm{T}_{2}$ effect may be negligible due to the use of small echo time offsets $\Delta \mathrm{TE}$, the $\mathrm{T}_{1}$ attenuation of individual lines may become significant from one end of the image to the other as typical numbers of lines and repetition intervals may lead to TM differences on the order of $400 \mathrm{~ms}$. However, the calculation of diffusion parameters is not affected except for the fact that reduced line intensities translate into correspondingly amplified noise in the diffusion maps.

\section{SNR Optimization}

Although the use of a long acquisition time $\mathrm{T}_{\text {acq }}$ for the individual STE signals reduces the receiver bandwidth and thereby image noise, it also prolongs the STE train and causes signal loss due to $\mathrm{T}_{2}$ signal attenuation (longer echo time $\mathrm{TE}_{\mathrm{STE}}$ with increasing number of slices $S$ ) and $\mathrm{T}_{1}$ relaxation (longer TM intervals with increasing number of lines $L$ ). Thus, the choice of an optimal acquisition time requires a trade-off between a low bandwidth for noise reduction and a short echo train for minimizing relaxation losses that depends on the FOV in line direction, volume coverage, and tissue relaxation times.

Assuming a fixed acquisition time for all echoes, the dependency of the SNR for line $l$ of slice $s$ on $\mathrm{T}_{\text {acq }}$ is given by

$$
\begin{gathered}
\operatorname{SNR}(l, s) \sim \sqrt{\mathrm{T}_{\mathrm{acq}}} \cdot \exp \left(-\frac{(l \cdot S-s) \cdot \mathrm{T}_{\mathrm{acq}}}{\mathrm{T}_{1}}\right) \\
\cdot \exp \left(-\frac{(2 s-1) \cdot \mathrm{T}_{\mathrm{acq}}}{\mathrm{T}_{2}}\right) .
\end{gathered}
$$

The three terms refer to the receiver bandwidth, the $\mathrm{T}_{1}$ attenuation during TM that increases with the echo train length, and the $\mathrm{T}_{2}$ attenuation during the preparation periods and the readout intervals. The influence of other parameters, such as the duration of the line-selective RF pulses and the time between the acquisition of consutive echoes, can be described by a constant factor in Eq. [1] which does not affect the determination of an optimized

$$
\mathrm{T}_{\mathrm{acq}}(l, s)=\frac{1}{2} \frac{\mathrm{T}_{1} \cdot \mathrm{T}_{2}}{(2 s-1) \cdot \mathrm{T}_{1}+(l \cdot S-s) \cdot \mathrm{T}_{2}} .
$$

Because the final line of the last slice has the lowest signal amplitude, a corresponding optimization results in

$$
\mathrm{T}_{\mathrm{acq}}(L, S)=\frac{1}{2} \frac{\mathrm{T}_{1} \cdot \mathrm{T}_{2}}{(2 S-1) \cdot \mathrm{T}_{1}+(L-1) \cdot S \cdot \mathrm{T}_{2}} .
$$

In the present study, the acquisition times were optimized for white matter, as it has the lowest signal intensity in single-shot LSI. Relaxation times of $\mathrm{T}_{1}=720 \mathrm{~ms}$ and $\mathrm{T}_{2}=70 \mathrm{~ms}$ were estimated from inversion recovery and SE experiments, respectively. Accordingly, for a single-slice LSI scan $(S=1)$ with $L=40$ lines Eq. [3] yields $\mathrm{T}_{\text {acq }}=7.304$ ms. As shown in Table 1, the experimentally used value of $7.296 \mathrm{~ms}$ slightly deviates from the optimal value to match a system-compatible sampling rate.

\section{Determination of Isotropic DW Images}

To avoid ambiguities in the interpretation of DW images of structurally heterogeneous tissues, it is advantageous, if not mandatory, to ensure an isotropic encoding of diffusion contrast independent of the relative directions of the applied gradients and the tissue components under investigation. Pertinent attempts need to assess the trace of the diffusion tensor, here defined as the apparent diffusion coefficient (ADC). It represents a weighted average of the diagonal elements $\mathrm{D}_{\mathrm{xx}}, \mathrm{D}_{\mathrm{yy}}$, and $\mathrm{D}_{\mathrm{zz}}$ according to

$$
\mathrm{ADC}=\frac{1}{3}\left(\mathrm{D}_{\mathrm{xx}}+\mathrm{D}_{\mathrm{yy}}+\mathrm{D}_{\mathrm{zz}}\right)
$$


Table 1

Experimental Parameters for DW Single-Shot LSI $\left(b=750 \mathrm{~s} \mathrm{~mm}^{-2}\right)$

\begin{tabular}{|c|c|c|c|c|}
\hline \# Slices & 1 & 1 & 3 & 7 \\
\hline TA & $466 \mathrm{~ms}$ & $512 \mathrm{~ms}$ & $485 \mathrm{~ms}$ & $463 \mathrm{~ms}$ \\
\hline $\mathrm{TE}_{\mathrm{SE}}$ & $57 \mathrm{~ms}$ & $57 \mathrm{~ms}$ & $57 \mathrm{~ms}$ & $57 \mathrm{~ms}$ \\
\hline \# Lines & 30 & 40 & 40 & 32 \\
\hline $\mathrm{FOV} / \mathrm{mm}^{2}$ & $90 \times 192$ & $120 \times 192$ & $120 \times 192$ & $120 \times 240$ \\
\hline Resolution $/ \mathrm{mm}^{2}$ & $1.5 \times 3.0$ & $3.0 \times 3.0$ & $3.0 \times 3.0$ & $3.75 \times 3.75$ \\
\hline Display $/ \mathrm{mm}^{2}$ & $1.5 \times 1.5$ & $1.5 \times 1.5$ & $1.5 \times 1.5$ & $1.9 \times 1.9$ \\
\hline Slice thickness & $6 \mathrm{~mm}$ & $6 \mathrm{~mm}$ & $8 \mathrm{~mm}$ & $8 \mathrm{~mm}$ \\
\hline Slice gap & - & - & $2 \mathrm{~mm}$ & $2 \mathrm{~mm}$ \\
\hline $\mathrm{TE}_{\mathrm{STE}}$ & $12.5 \mathrm{~ms}$ & $10.5 \mathrm{~ms}$ & $5.4 \mathrm{~ms}$ & $4.1 \mathrm{~ms}$ \\
\hline$\Delta \mathrm{TE}$ & - & - & $4.36 \mathrm{~ms}$ & $2.44 \mathrm{~ms}$ \\
\hline $\mathrm{T}_{\mathrm{acq}}$ & $9.216 \mathrm{~ms}$ & $7.296 \mathrm{~ms}$ & $2.176 \mathrm{~ms}$ & $1.216 \mathrm{~ms}^{1}$ \\
\hline TR & $12.4 \mathrm{~ms}$ & $10.5 \mathrm{~ms}$ & $9.8 \mathrm{~ms}$ & $11.5 \mathrm{~ms}$ \\
\hline $\mathrm{SNR}^{2}$ & 58 & 109 & 82 & 90 \\
\hline
\end{tabular}

TA: acquisition time, FOV: field-of-view, TE offset, $\mathrm{T}_{\text {acq }}$ : acquisition time per echo, TR: line repetition time.

${ }^{1}$ Not optimized according to Eq. [3] because of gradient limitations.

${ }^{2}$ Central slice of a water phantom $(b=0)$.

The calculation of an isotropic DW image and/or an ADC map was based on four independent single-shot LSI measurements, i.e., one scan without diffusion gradients and three differently DW scans. The strategy used follows a proposal for an efficient gradient scheme (15) that applies combinations of three orthogonal gradients with amplitude ratios along the read:line:slice directions of $1: \frac{1}{2}: \overline{1}$ for $D_{x x}$, $1: \overline{1}: \frac{1}{2}$ for $D_{y y}$, and $\frac{1}{2}: \overline{1}: \overline{1}$ for $D_{z z}$ in successive experiments. The diagonal elements are calculated from

$$
\mathrm{D}=\frac{1}{b} \ln \left(I_{0} / I\right)
$$

with $I_{0}$ and $I$ the LSI signal intensities without and with diffusion encoding, respectively, and a weighting factor $b$ given by

$$
b=\gamma^{2} g^{2} \delta^{2}\left(\Delta-\frac{\delta}{3}\right)
$$

with $\gamma$ the gyromagnetic ratio, $g$ the diffusion gradient amplitude, $\delta$ the gradient duration, and $\Delta$ the diffusion time, i.e., the period between the dephasing and rephasing gradient lobes. Here, DW images were obtained with $b=$ $750 \mathrm{~s} \mathrm{~mm}^{-2}$ based on $g=18.4 \mathrm{mT} \mathrm{m}^{-1}, \delta=25.2 \mathrm{~ms}$, and $\Delta=$ $30.1 \mathrm{~ms}$. With these parameters the echo time of the SE preparation period was $\mathrm{TE}_{\mathrm{SE}}=57 \mathrm{~ms}$.

\section{DW Single-Shot LSI of the Human Brain}

All studies were performed at $2.0 \mathrm{~T}$ using a whole-body MRI system (Magnetom Vision, Siemens, Erlangen, Germany) with $25 \mathrm{mT} \mathrm{m}^{-1}$ gradients and a maximum slew rate of $40 \mathrm{~T} \mathrm{~m}^{-1} \mathrm{~s}^{-1}$. Images were obtained with the use of the standard head coil. Conventional data processing for Fourier imaging was adapted to LSI by discarding the Fourier transformation along the direction of the moving lines, i.e., in the direction of the usual 2D phase-encoding gradient, and replacing the normal sinc-interpolation (i.e., zero- filling) by a linear interpolation of intensities from directly neighboring lines. An on-line algorithm for a pixel-bypixel calculation of an ADC map and an isotropic DW image from four independent acquisitions (see above) was added to the image reconstruction software of the scanner.

Typical imaging parameters for DW single-shot LSI sequences using 30-40 lines and 1-7 slices are summarized in Table 1. Measurements were carried out on phantoms and healthy adults. Written informed consent was obtained in all cases before the examinations. Neither cardiac gating nor special head restraints were used. Transverse single-shot LSI of the brain was performed by exciting lines from right to left and multiple slices from head to feet. In single-slice sagittal images, the lines were acquired from head to feet or posterior to anterior, depending on the desired FOV.

\section{RESULTS AND DISCUSSION}

Studies of a water phantom were performed to adjust the diffusion sensitivity of the DW single-shot LSI method and to assess its accuracy in determining quantitative diffusion coefficients. A single slice was measured with a resolution of $3.0 \times 3.0 \mathrm{~mm}^{2}$ (for other parameters see Table 1). To investigate the influence of partial saturation on the ADC calculated from four DW scans, the delay time between successive measurements was varied from $12 \mathrm{~s}$ (full relaxation) to $1 \mathrm{~s}$ (significant saturation). In all cases, the diffusion coefficients were in excellent agreement with the

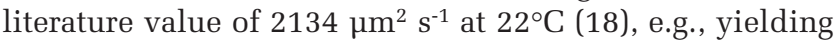
$2105 \pm 23 \mu \mathrm{m}^{2} \mathrm{~s}^{-1}$ for an inter-scan delay of $12 \mathrm{~s}$ and $2106 \pm$ $61 \mu \mathrm{m}^{2} \mathrm{~s}^{-1}$ for a $1 \mathrm{~s}$ delay. The larger standard deviation in the case of the shorter delay reflects the signal loss due to saturation when using the same number of scans.

\section{DW Single-Shot LSI of the Human Brain: Single Slices}

The upper four images in Figure 2 depict single-shot LSI scans (Fig. 2a) without diffusion gradients and (Fig. 2b-d) 

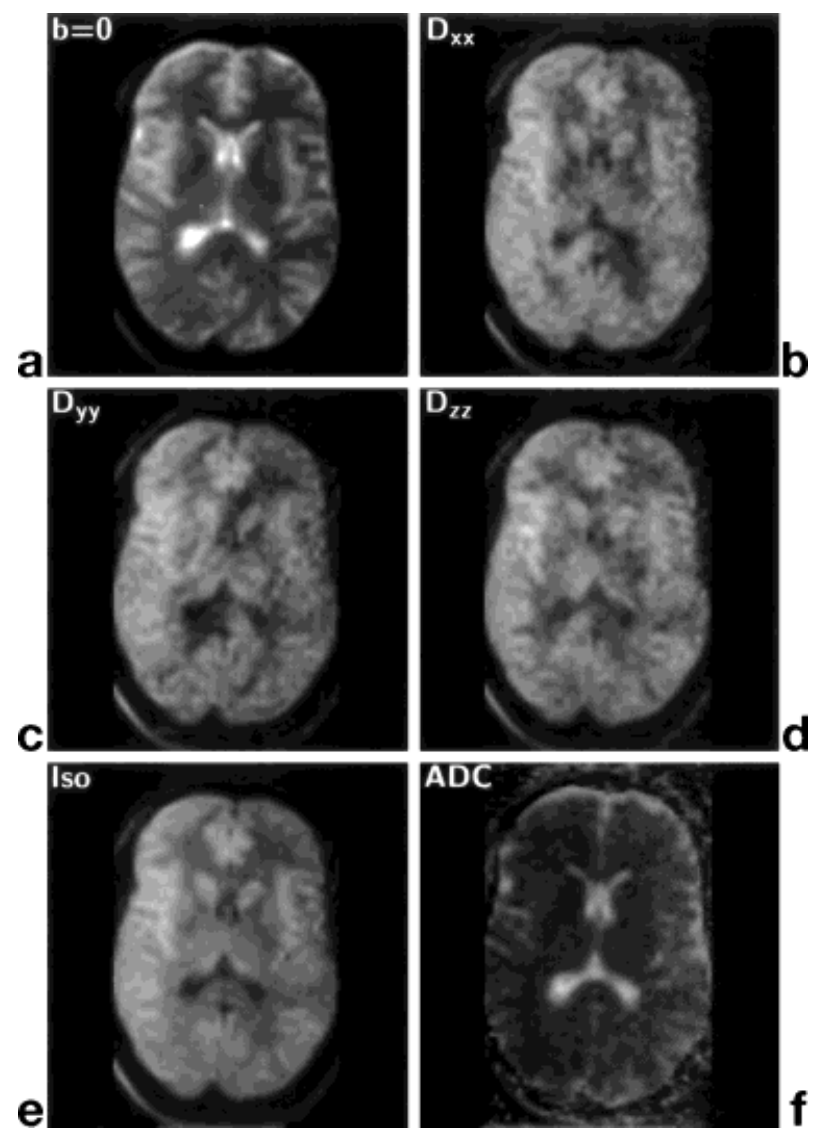

FIG. 2. Single-shot LSI of the human brain (a) without DW $(b=0)$ and (b-d) with DW ( $\left.b=750 \mathrm{~s} \mathrm{~mm}^{-2}\right)$. The images represent single-slice acquisitions with an imaging time of $512 \mathrm{~ms}$ and a resolution of $3.0 \times 3.0 \mathrm{~mm}^{2}$ interpolated to $1.5 \times 1.5 \mathrm{~mm}^{2}$ (for other parameters see Table 1). The relative amplitudes of the diffusion gradients (line:read:slice) were $1: \frac{1}{2}: \overline{1}$ for $D_{x x}$ in (b), $1: \overline{1}: \frac{1}{2}$ for $D_{y y}$ in (c), and $\frac{1}{2}: \overline{1}: \overline{1}$ for $D_{z z}$ in (d). (e) Calculated isotropic DW image $(b=750 \mathrm{~s}$ $\mathrm{mm}^{-2}$ ), and (f) ADC map. The total measurement time was $20 \mathrm{~s}$ (four DW scans, inter-scan delay $6 \mathrm{~s}$ ).

with diffusion encodings that reflect the diagonal elements of the diffusion tensor. Each image was obtained within $512 \mathrm{~ms}$ and at $3.0 \times 3.0 \mathrm{~mm}^{2}$ resolution (for other parameters see Table 1). The full set of four images required a measuring time of $20 \mathrm{~s}$ for an inter-scan delay of $6 \mathrm{~s}$. The dependence of the diffusion contrast on the gradient orientation is clearly visible, for example, in the splenium of the corpus callosum and in the posterior limb of the internal capsule. However, because the diffusion encoding gradients are not along the image axes, but point along oblique directions, the resulting signal attenuation due to diffusion weighting is not symmetric.

In agreement with the general properties of single-shot LSI (17), the images exhibit a mild signal intensity decrease from left to right that follows the excitation order of the lines and reflects their increasing $\mathrm{T}_{1}$ attenuation. Figure 2e,f shows an isotropic DW image calculated for the same $b$ factor as used for Fig. 2b-d and an ADC map, respectively.

DW single-shot LSI sequences do not depend on the availability of EPI-capable gradients with slew rates greater than $100 \mathrm{~T} \mathrm{~m}^{-1} \mathrm{~s}^{-1}$. In fact, a restriction of the gradient amplitude to $15 \mathrm{mT} \mathrm{m}^{-1}$ and of the ramp time to $1.0 \mathrm{~ms}$, i.e., a slew rate of $15 \mathrm{~T} \mathrm{~m}^{-1} \mathrm{~s}^{-1}$, prolongs the measurement time for the LSI scans in Fig. 2 by less than 100 ms and causes only mild consequences for the SNR due to increased TE and TM intervals. For white matter, the signal of the last image line is estimated to be reduced by $13 \%$.

The images in Figure 3 refer to transverse and sagittal brain stions with a resolution of $1.5 \times 3.0 \mathrm{~mm}^{2}$. They represent single-shot images obtained within a measuring time of $466 \mathrm{~ms}$ without diffusion gradients (upper row) and with diffusion encoding along one direction (middle row). Figure 3 also shows the corresponding isotropic DW images calculated from a full set of four scans as in Fig. 2e (bottom row).

Most importantly, the images in Fig. 3 demonstrate two advantageous features of single-shot LSI using stimulated echoes. First, inner volume scans that cover only a limited
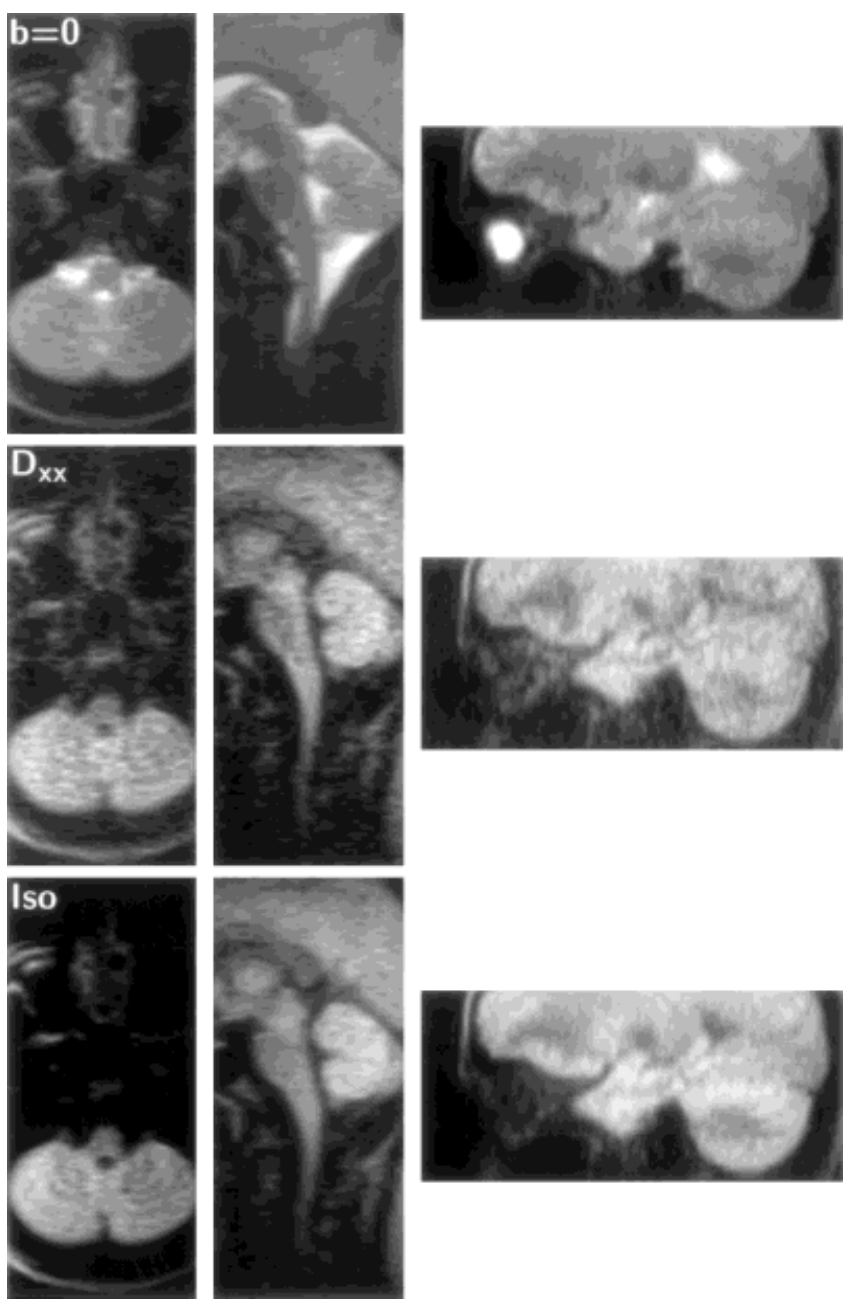

FIG. 3. Inner volume DW single-shot LSI of the human brain in the presence of magnetic field inhomogeneities. The images represent single-slice acquisitions with an imaging time of $466 \mathrm{~ms}$ and a resolution of $1.5 \times 3.0 \mathrm{~mm}^{2}$ interpolated to $1.5 \times 1.5 \mathrm{~mm}^{2}$ (for other parameters see Table 1). (Upper row) LSI scans without DW $(b=0)$ and (middle row) with DW ( $b=750 \mathrm{~s} \mathrm{~mm}^{-2}, 1: \frac{1}{2}: 1$ for $\left.D_{\mathrm{xx}}\right)$, (bottom row) isotropic DW images $\left(b=750 \mathrm{~s} \mathrm{~mm}^{-2}\right)$. LSI scans of a small inner FOV are free from aliasing artifacts (no phase-encoding) and do not suffer from air-tissue susceptibility differences (RF refocused echoes). 

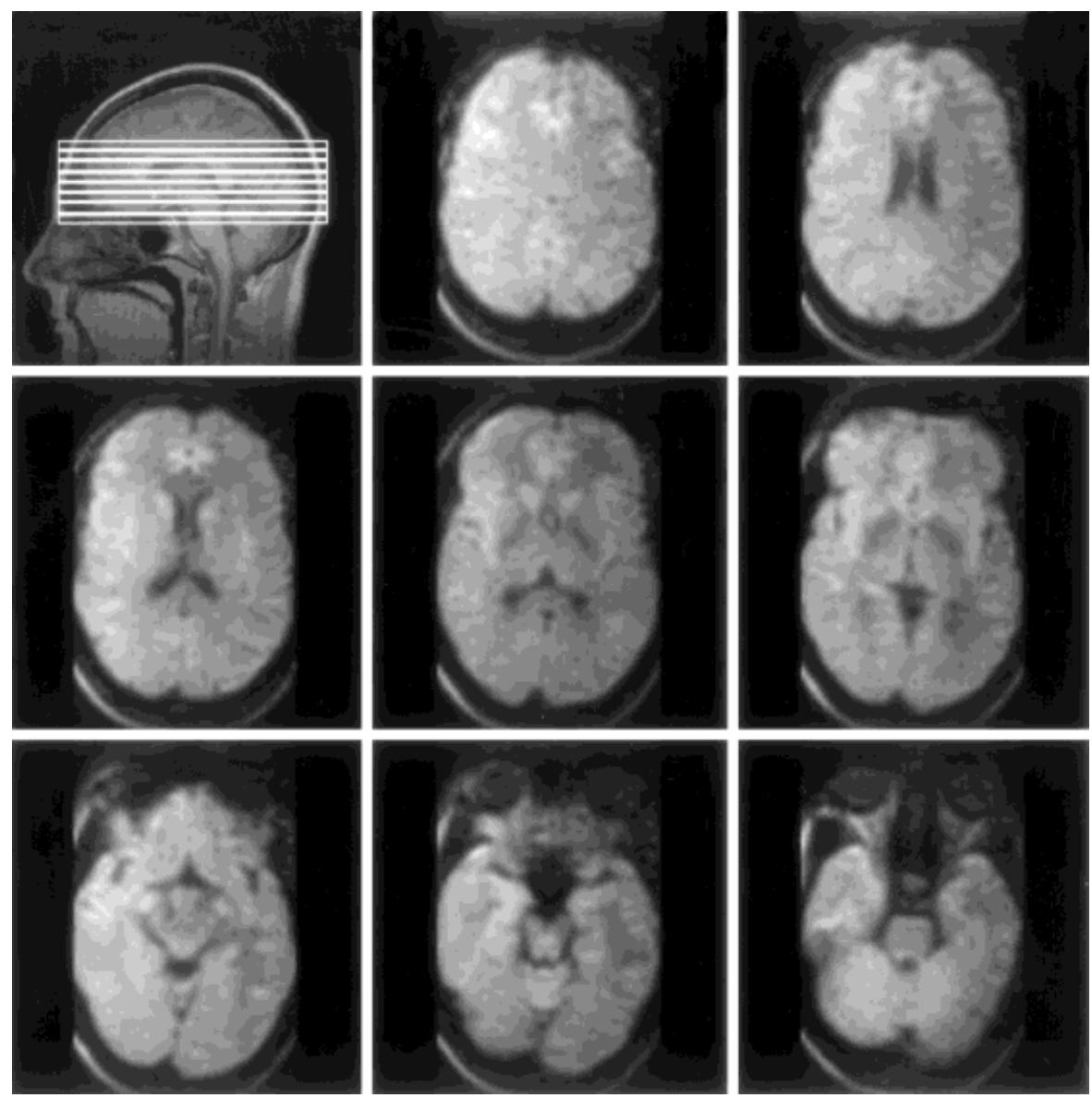

FIG. 4. Sequential multi-slice DW LSI of the human brain. Sagittal scout image and eight isotropic DW images $(b=750 \mathrm{~s}$ $\mathrm{mm}^{-2}$ ) depicting transverse sections each obtained from four DW single-shot singleslice LSI scans (from top left to lower right, $1.5 \mathrm{~mm}$ gap). The $3.0 \times 3.0 \mathrm{~mm}^{2}$ resolution was interpolated to $1.5 \times 1.5$ $\mathrm{mm}^{2}$ (for other parameters see Table 1). The total measurement time was $78 \mathrm{~s}$ (four DW scans per slice, 2-s inter-scan delay).
FOV are free from aliasing artifacts because of the absence of Fourier phase encoding. In technical terms, the reduction of the FOV, i.e., the use of a lower number of lines, either allows for a longer $\mathrm{T}_{\mathrm{acq}}$ (smaller receiver bandwidth) or a shorter imaging time. Second, in contrast to EPI, single-shot LSI is not at all hampered by the presence of air-tissue susceptibility differences. This is because the acquired STE signals represent RF refocused echoes that are insensitive to magnetic field inhomogeneities and resonance offsets. Corresponding EPI scans of the sections selected for Fig. 3 are severely corrupted by geometric distortions and suffer significant regional signal loss.

\section{Multi-Slice Capabilities}

The extension of DW single-shot LSI to multi-slice acquisitions requires some consideration because even singleslice measurements excite large portions of an object along all three dimensions. Thus, repetitive measurements need a relaxation recovery period and sequential multi-slice measurements of single-shot LSI scans cannot be performed at arbitrary speed. Experimental studies demonstrated that a 2-s inter-scan delay provides a reasonable compromise between a sufficiently short overall measurement time and an SNR that is only mildly affected by saturation. In this case, steady-state preparation scans precede only the first slice as subsequent LSI sequences continue to "prepare" respective magnetizations by their inherent volume excitations. Figure 4 depicts the isotropic
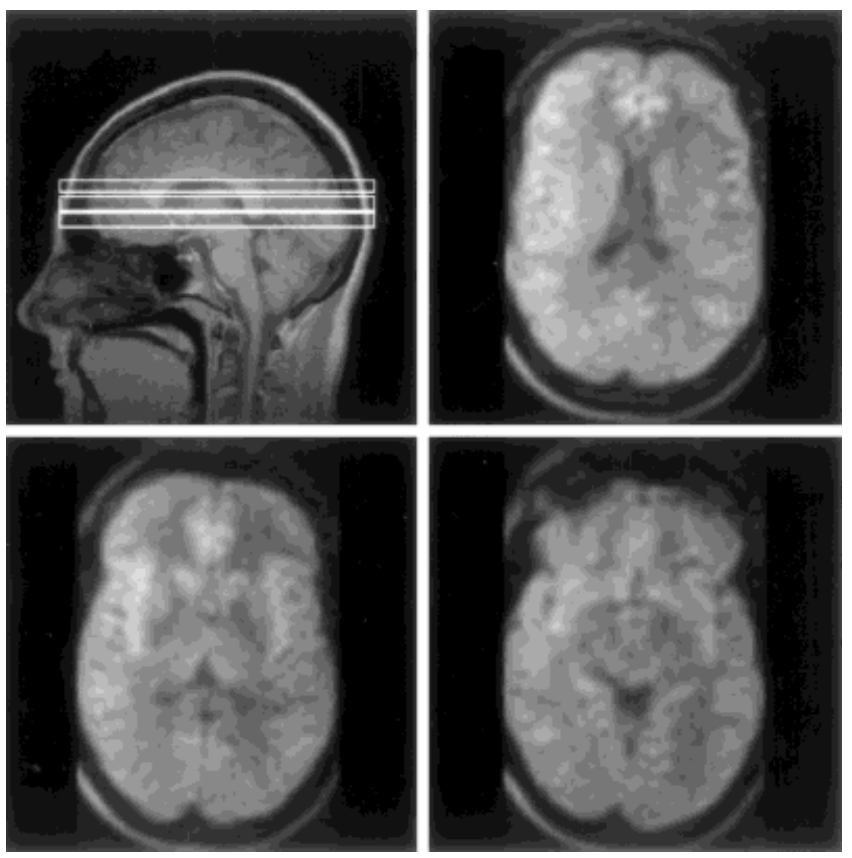

FIG. 5. Single-shot multi-slice DW LSI of the human brain. Sagittal scout image and three isotropic DW images $(b=750 \mathrm{~s} \mathrm{~mm}$ ) depicting transverse sections each obtained from four DW singleshot multi-slice LSI scans. The resolution of $3.0 \times 3.0 \mathrm{~mm}^{2}$ was interpolated to $1.5 \times 1.5 \mathrm{~mm}^{2}$ (for other parameters see Table 1). The total measurement time was $20 \mathrm{~s}$ (four DW scans, inter-scan delay $6 \mathrm{~s}$ ). 
DW images of eight slices from a sequential acquisition of a corresponding set of DW single-shot LSI scans obtained within a total measurement time of $78 \mathrm{~s}$.

A more time-efficient coverage of larger volumes should be achievable by exploiting the true multi-slice capability of the single-shot LSI sequence, as indicated in Fig. 1. However, such applications lead to a compromise between the number of simultaneously acquired slices and the individual image quality in terms of spatial resolution and SNR. Figures 5 and 6 show the results from a single-shot 3-slice and 7-slice LSI acquisition, respectively, as a demonstration of the quality of respective multi-slice isotropic DW images. In both cases, the overall measurement time
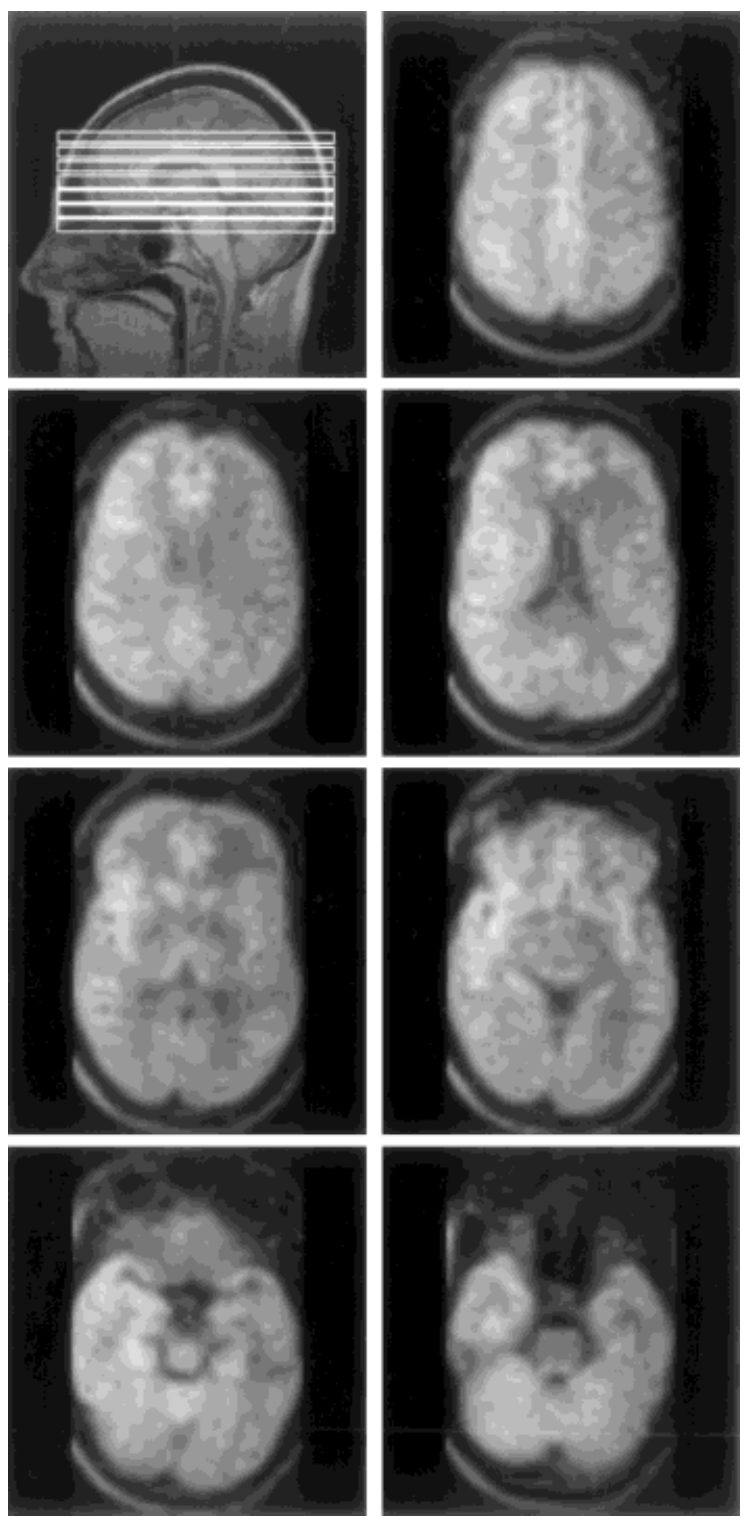

FIG. 6. Single-shot multi-slice DW LSI of the human brain. Sagittal scout image and seven isotropic DW images $\left(b=750 \mathrm{~s} \mathrm{~mm} \mathrm{~mm}^{-2}\right)$ depicting transverse stions each obtained from four DW single-shot multi-slice LSI scans. The resolution of $3.75 \times 3.75 \mathrm{~mm}^{2}$ was interpolated to $1.9 \times 1.9 \mathrm{~mm}^{2}$ (for other parameters see Table 1). The total measurement time was $20 \mathrm{~s}$ (four DW scans, inter-scan delay $6 \mathrm{~s})$. was 20 s using a 6-s delay for the four DW scans. The measurement time reduces to $8 \mathrm{~s}$ for a 2-s delay, as in Fig. 4.

\section{CONCLUSION}

Single-shot LSI using stimulated echoes was adapted to multi-slice DW MRI and applied to isotropic diffusion mapping of the human brain on a conventional $2.0 \mathrm{~T}$ whole-body scanner. The method offers subsecond imaging times and proves to be insensitive to motion as a major prerequisite for a reliable diffusion study. In addition, because single-shot LSI sequences employ RF refocused signals, the resulting images suffer from neither geometric distortions or signal losses due to tissue susceptibility differences nor ghosting artifacts from water and fat contributions. Of course, DW single-shot LSI may be easily extended to full diffusion tensor imaging.

The main limitation for DW single-shot LSI is the low SNR. Two factors contribute to this problem: first, the SNR of LSI is reduced by the square root of the number of lines compared to 2D Fourier imaging. Second, an STE yields only half the signal strength of a comparable SE. In comparison to EPI, some compensation is achieved by the smaller receiver bandwidth of single-shot LSI sequences because the overall length of the data acquisition period is only restricted by $\mathrm{T}_{1}$ attenuation rather than by the much faster $\mathrm{T}_{2}^{*}$ decay as for EPI. Another difference to EPI is the restricted multi-slice capability. Whereas single-shot multislice LSI acquires multiple slices at the expense of spatial resolution in a single acquisition, sequential single-shot LSI scans from multiple slices require an inter-scan recovery period of about 1-2 s. In contrast to EPI, DW single-shot LSI benefits from high magnetic fields due to its insensitivity to susceptibility artifacts and the fact that the prolonged $\mathrm{T}_{1}$ relaxation times allow the use of longer acquisition periods.

Whether DW single-shot LSI reaches general diagnostic quality or only helps to replace DW EPI in brain regions contaminated by magnetic field inhomogeneities remains to be seen in future studies.

\section{REFERENCES}

1. Maudsley ME, Cohen Y, Mintorovitch J, Chileuitt L, Shimizu H, Kucharczyk J, Wendland MF, Weinstein PR. Early detection of regional cerebral ischemia in cats: comparison of diffusion- and $\mathrm{T}_{2}$-weighted MRI and spectroscopy. Magn Reson Med 1990;14:330-346.

2. Warach S, Chien D, Li W, Ronthal M, Edelman RR. Fast magnetic resonance diffusion-weighted imaging of acute human stroke. Neurology 1992;42:1717-1723.

3. Turner R, Le Bihan D, Maier J, Vavrek R, Hedges LK, Pekar J. Echoplanar imaging of intravoxel incoherent motion. Radiology 1990;177: 407-411.

4. Haase A. Snapshot FLASH MRI. Application to $\mathrm{T}_{1}, \mathrm{~T}_{2}$, and chemicalshift imaging. Magn Reson Med 1990;13:77-89.

5. Frahm J, Haase A, Matthaei D, Merboldt KD, Hänicke W. Rapid NMR imaging using stimulated echoes. J Magn Reson 1985;65:130-135.

6. Hennig J, Nauerth A, Friedburg H. RARE imaging: a fast imaging method for clinical MR. Magn Reson Med 1986;3:823-833.

7. Merboldt KD, Hänicke W, Bruhn H, Gyngell M, Frahm J. Diffusion imaging of the human brain in vivo using high-speed STEAM MRI. Magn Reson Med 1992;23:179-192.

8. Deimling M, Müller E, Laub G. Diffusion-weighted imaging with turboFLASH. In: Proc. SMRM, 9th Annual Meeting, New York, 1990. p 387. 
9. Perman WH, Gado M, Sandstorm JC. DPSF: Snapshot FLASH diffusion/ perfusion imaging. In: Proc. SMRM, 9th Annual Meeting, New York, 1990. p 309.

10. Norris DG, Börnert P, Reese T, Leibfritz D. On the application of ultra-fast RARE experiments. Magn Reson Med 1992;27:142-164.

11. Schick F. SPLICE: Subsecond diffusion-sensitive MR imaging using a modified fast spin-echo acquisition mode. Magn Reson Med 1997;38: 638-644.

12. Crooks LE. Selective irradiation line scan techniques for NMR imaging. IEEE Trans Nucl Sci 1980;27:1239-1244.

13. Maudsley AA. Multiple-line-scanning spin density imaging. J Magn Reson 1980;41:112-126.
14. Ailion DC, Ganesan K, Case TA, Christman RA. Rapid line scan technique for artifact-free images of moving objects. Magn Reson Imag 1992;10:747-754.

15. Gudbjartsson H, Maier SE, Mulkern RV, Mórocz IÁ, Patz S, Jolesz FA. Line scan diffusion imaging. Magn Reson Med 1996;36:509-519.

16. Gudbjartsson H, Maier SE, Jolesz FA. Double line scan diffusion imaging. Magn Reson Med 1997;38:101-109.

17. Finsterbusch J, Frahm J. Single-shot line scan imaging using stimulated echoes. J Magn Reson 1999;137:144-153.

18. Weingärtner H. Self diffusion in water. A reassessment. Zeitschr Physikal Chemie 1982;132:129-149. 